\title{
ON TEXT COHERENCE PARSING
}

\author{
Udo Iahn \\ Alben-Ludwigs-Universităt L'reiburg \\ Linguistische Informatik / Computerlinguistik \\ Friedrichstr. 50 \\ D-W-7800 Freiburg i. Brsg. \\ Germany \\ email: hahn@supreme.coling.uni-freiburg.de
}

\begin{abstract}
ABSTKACT
In this paper global patterns of thematic text organization are considered within the framework of a clistributed model of text understanding. Hased on the parsing results of prior text cohesion analysis, specialized text graminar ntodules deternine whether some well-defined text macro-organization jattern is compuable from the available text representation structures. The model underlying text colecrence parsing formalizes hitherto entirely intuitive textlinguistic notions whose origin can be traced back to Danes's work on thematic progression patterns.
\end{abstract}

\section{INTRODUCTION}

During the last years it has become incicasingly apparent that dialog and 1ext understanding systems must account for connectivity relations thit extend over sentence boundaries. This has led to at bulk of work dealing with various forms of cohesion-preserving language mechanisms, mainly in the ficld of anaphora, which contribute to connectivity among sentences. From the focus on these linguistic phenomena one might obtain a misleading picture of textual connectivity, viz. one that considers it basically as at 'flat', continuous stream of formally connected utterances lacking additional structure. Far less research has been devoted to the internal organization of cohesive utterances by mechanisms at a more global level of dialog/text architecture, the level of text coherence.

Major computational approaches related to co. herence aspects within a dialog processing framework are duc to Reichman's [1978], McKeown's [1985] and Scha \& Polanyi's [1988] fornalizations of dialog gratnmars. Coherence criteria of written texts have teen investigated in the context of 'Rhetorical Structure Theory' [Mann \& 'Thompson 1988] and related extensions [c.g., Alterman 1982, T'ucker, Nirenburg \& Raskin 1986] of the originat theory of coherence relations in discourse [Hobbs 1982]. A sccond major methodology which deals with the global stuucturing of written texts is the model of text macro propositions and superstructurcs [Kintsch \& van Dijk 1978, van Dijk 1980), the later sharing all relevant properties one generally attributes to story grammars [Rumelhari 1975]. The problem with this kind of methodology is that, unlike the coherence relation approatch, the grammats which hatve been proposed so far are fatirly idiosyncratic for each applicattion domain (narratives, weallher reports, etc.). Common to all these approaches is the requirement of a decp, propositionally guided understanding of the stiderlying discourse; in particular, a complete theory of its donain and an exhaustive specification of a natural language grammar must be supplied in order to guarantee proper operation of implemented systems. This might explain why, with only few exceptions, these models of text coherence tave resisted furthe conputational treatment as evidenced by operational sysiems.

We here make an alternative and compotation nally more tractable proposal on how to deal with global text strictures at the text coherence level. Its roots can be traced back to the seminal work of 1 . Dines [1974], in which be informally developed the notion of thematic progression patterns, distinguisting between three prototypical pattenis, vi\%. constant thene, continuous thematization of rhenes, and derived ilsene (see section 3). 'The model outlined in this paper statis from a thorough formalization of (one of) these notions and places it into the environment of a fully operational text parsing system whose design is mainly oriented towards the proper recogrnition of text cohesion and coherence phe nomena. Pertinent reasons for our choice of a Danestyje model of text coherence ate:

(1) The text parser fonns part of the text undestanding system Tol'le. It operates it at real world domain [Reiner \& thatu 1988], i.e. textual input is taken from a pemancht strearn of test reporis in major German in formation technology magazines. As it seems that it will remain infeasible for a long time to cone to provide exhaustive domain and granmar specifications for rontinely operating text understanders, a patticularly robust partial parsing appooach capable of handling potential specification gaps has been adopred. These conditions obviously preclude the consideration of RST'-style co herence relation computing as a text coherence analysis stategy, since relevant knowledge portions might be lacking for determining specific instances of colerence relations. Conversely, the coherence relation approach scens currently infeasible for the routine processing, of large-scale text collections in real donains.

(2) The description of coherenee structures in terms of coherence relations or text macro propositions requires the availability of deep assertional knowledge from theis application domain (A-box level specifications in Krypton terminology; of. Brachman et al. [1985|). The TOP' IC system, towever, emphasizes the role of terminelogical knowledge of its domain, i.e. the description of prototypical projerties and inlerence rules related to basic conceptual units of the domain (Krypton's T- $-(x) x$ level knowledge). As TOPIC is rather weak with respect to full-blown assertional knowledge, coherence relation computing, however valuable it might be, is currently out of reach for this system. Fortunately, Danes-type coherence patterns primarily efere to the level of teminological knowledge.

(3) Prototypical pattens of thematic progression ate fairly general and independent of particular domains that expository texts deal with. Lingustic studies have 
collected empirical evidence for this claim through investigations of texts from diverse domains [Giora 1983a, Kurzon 1984]. This coincides with the generality of use of most coherence relations, but is in sharp contrast to the highly constrained and domain-dependent model of superstructures and story grammars.

(4) Major thematic progression patterns are correlated with particular search styles and retrieval modes in fulltext information systems. Hence, providing typed coherence operators inherently supports graphics-based user interactions with the TOPIC system in terms of advanced conceptual orientation and navigation tools for semantically guided text graph tours (see section 5.3).

(5) The investigation of thematic progression pattems is of value in its own methodological right. They constitute a basic structural model of text macro organization as opposed to model-theorctic and plan/goal-based approaches (a distinction made by Pustejovsky [1987]). As such they might complement current text understanding methodologies whose emphasis, so far, has bcen on fairly knowledge-expensive assertional models (such as coherence relations and text macro propositions) or stereotyped text-semantical models (such as superstructures and story grammars).

\section{MOTIVATING THE NEED FOR TEXT COHERENCE PARSING}

The model of text structure parsing we propose draws a careful distinction between text cohesion and text coherence phenomena. As to the illustration of text cohesion mechanisms in natural language texts, consider the following text passage:

[1] The Delta-X from ZetaMachines Inc. is a computer system that runs Unix V.3.

[2] The system is based on a 68020 processor.

[3] It has a 12-inch monochrome display and an integrated telephone handset and built-in modem.

[4] Intemally, there's a 40-megabyte hard disk, a 1.2megabyte 51/4-inch floppy disk drive, 4.5 megabytes of RAM, three $R S-232 C$ ports, and an ST-506 port.

Repeated occurrences of various text cohesion phenomena are illustrated by nominal anaphora ("The system" in [2]), pronominal anaphora (' $I t^{\prime}$ in [3]), both referring to the unique antecedent Delta-X (in [1]), while 'Internally, there's a ... hard disk' (in [4]) is linked to $D e l t a-X$ via textual ellipsis. The basic cohesion among these sentences yields the common thematic background for constantly elaborating on a single topic (Delta-X), An appropriate text parser should, first of all, recognize these multiple cohesion phenomena and produce something like the following representation structures (indicated by $[\ldots]_{R}$ ):

\footnotetext{
$[1]_{R}$ Delta-X < manufacturer: $\{$ ZetaMachines Ine. $\}>$

Delta $-X<$ operating systern: $\left\{U_{n i x} V_{.3}\right\}>$

$[2]_{R}$ Delia-X <CPU: $\{68020\}>$

$13\}_{R}$ Deltu $\mathrm{X}<$ peripheral devices: $\{12$-inch monochrome display $\}>$

Delta $\mathrm{X}<$ peripheral devices: [ telephone handset ] >

Delta- $X<$ communication devices: ( modem ) >

$[4]_{R}$ Delta-X $<$ external storage devices: $[40$-megabyce hard disk ] > Delta-X < extemal storage devices:

[ 1.2-megabyte 51/4-inch floppy disk drive ]

Delta- $X<$ main memory: $\{4.5$ megabytes of RAM $\}>$

Delta-X $<$ ports: $\{3$ RS-232C poris $\}>$

Delis-X < ports: \{ ST-506 port \}>
}

ACIYS DE COLING-92, NANTES, 23-28 AÔ̄T 1992
What is still lacking is a representation facility which characterizes this sequence of single assertions constantly referring to a single topic (Delta-X) as constituting a coherent whole. Recognizing linguistic forms of text coherency and providing appropriate thematic grouping operators for text knowledge bases is what text coherence parsing mainly is about. Even if parsers would perfectly recognize and normalize all occurrences of text cohesion phenomena in texts, missing recognition capabilities for text coherence phenomena would nevertheless produce under-structured, incoherent text knowledge bases in the sense that global pragmatic indicators of discourse bracketing would be lacking.

\section{BASIC TEX'T COHERENCE PATTERNS}

In this section, we informally describe the basic patterns of text coherence focused on in this paper. According to Danes [1974] three categories of thematic developments can be distinguished:

Constant Theme. This pattem is characterized by the constant elaboration of one specific topic within a text (passage) by considering several of its conceptual facets. The following two paragraphs serve to illustrate this major pattern of thematic progression (the reference points to the constant theme (Delta$X)$ are indicated by italics):

[T1.1]. The Delta-X from ZetaMachines Inc. is a multiuser, multitasking computer system that runs Unix V.3 and comes complete with most of the software needed for business applications. The combination host computer/workstation is based on a 68020 processor, with dual 68000 processors providing peripheral processing. It has a 12-inch monochrome display and an integrated telephone handset and built-in modem.

Internally, there's a 40-megabyte hard disk, a 1.2megabyte 51/4-inch floppy disk drive, 4.5 megabytes of $R A M$, a network controller, three $R S-232 C$ ports, and an ST-506 port.

$\square$ Continuous Thematization of Rhemes. In contrast to constant themes, this pattern realizes a continuous shift of topics (visualized by bold italics). The process starts with a theme and some comment on that theme which we shall call rheme (actually, an elaboration on one of its conceptual facets). Now this rheme is focused on as the next theme that is claborated by a corresponding rheme, etc.:

[T1 2]. The $\$ 12,000$ Delta-X host/workstation can be supplied from ZetaMachines Inc., 2999 Statc St., Santa Barbara, CA 93105. Zeta-Machines' sales manager, Brian Wilson, says that they also plan to market the Gamma-Z, a CAD/CAM workstation based on a Connection Machine architecture. The underlying theoretical foundations are due to $D$. Hillis, a former M.I.T. student who first developed an experimental prototype based on connectionist principles.

$\square$ Derived Theme. Global text structurc can also bc introduced by a variety of topics which share conceptual commonalities (facets) at the knowledge representation level (not necessarily need this be paralleled with properties actually mentioned in the text!) without the general concept being explicitly stated in the text. Technically this is realized by a set of sub- 
ordinates or instances of a common (only implicit) superordinate/prototype. Suppose the illustrative text [T1] composed of its two constituent parts from above, [ T 1.1] and [T 1.2], is augmented by several paragraphs dealing with Gamma-Z and Sigma-P machines on a similar level of detail as those passages which consider the Delta-X in [T1]:

['T2]. The Delta-X from ZetaMachines... [T' 1.1由T11.2] The Gamma-Z is a $M S \cdot D O S$ machinic. Peripheral devices include an 8 -inch color display, a matrix printer, and a keyboard....

The Sigma-P system makes available a lot of desirable application software such as a databuse system, word processing, and a variciy of games. ...

This text implicitly has workstation as a derived theme, since that is the immediate prototype concept of those three instances (Delta-X, Gamma-Z, Sigma-P) explicitly mentioned in [T 2$]$.

\section{THE KNOWLEDGE SOURCES INVOLVED IN TEXT PARSING}

This section deals with the knowledge sources involved in actually parsing a text. Basically (see Figure 1), thesc are constituted by the PARSE BULLETIN, a blackboard-type memory which records the single events of the parsing process, the DOMAIN KNOWLEDGE BASE, which contains the domain-specific background knowledge needed for the parse, and various EXPERT for actually driving the parse through the text grammar specifications they incorporate (cf. Hahn [1990] for a more comprehensive presentation).

The PARSE BULLETIN has a flat list struc. turc. It records the sequence of text tokens as they appear in the text and, if relevant (see below), notes their class identifiers (FRAME item, ADJcctive, etc.). More important, constructive parsing activities based on operations of the knowledge base and the parser are indicated at several positions (so-called parse points) in the PARSE BULLETIN. The type of operation being performed is indicated by a particular parse descriptor. Some are intemal to the management of the knowledge base, c.g.. DEFACT (default concept activation), while others indicate grammatical relations recognized by the parscr, such as NounATT (conceptual attribution relations between nouns), AdjATT (conceptual attribution relations between adjectives and nouns). The items affected by an operation form a so-called parse tuple.

The parser does not consider every token it receives from the input text at the same level of detail. Instead, it distinguishes twetween words which are significant to its performance (conceptually relevant ones, such as nouns or adjectives which denote concepts in the domain knowledge base, or linguistically refevant ones, such as negation particles, certain conjunctions, quantifiers, etc.), and those that are not (anong them a wide variety of semantically indifferent nouns, verbs, particles, etc., each of which is assigned the class identilier NIL). The latter are simply discarded from further analysis, while the former are assigned lexicalized grammar sfecifications. The parser has thus been turied towards partial parsing in a spirit similar to that advocated by Schank et al. [1980] and achieves text understanding primarily on a terminological level of knowledge representation.

ACLLS J): COLING-92, NANITS, 23-28 AOŨ' 1992

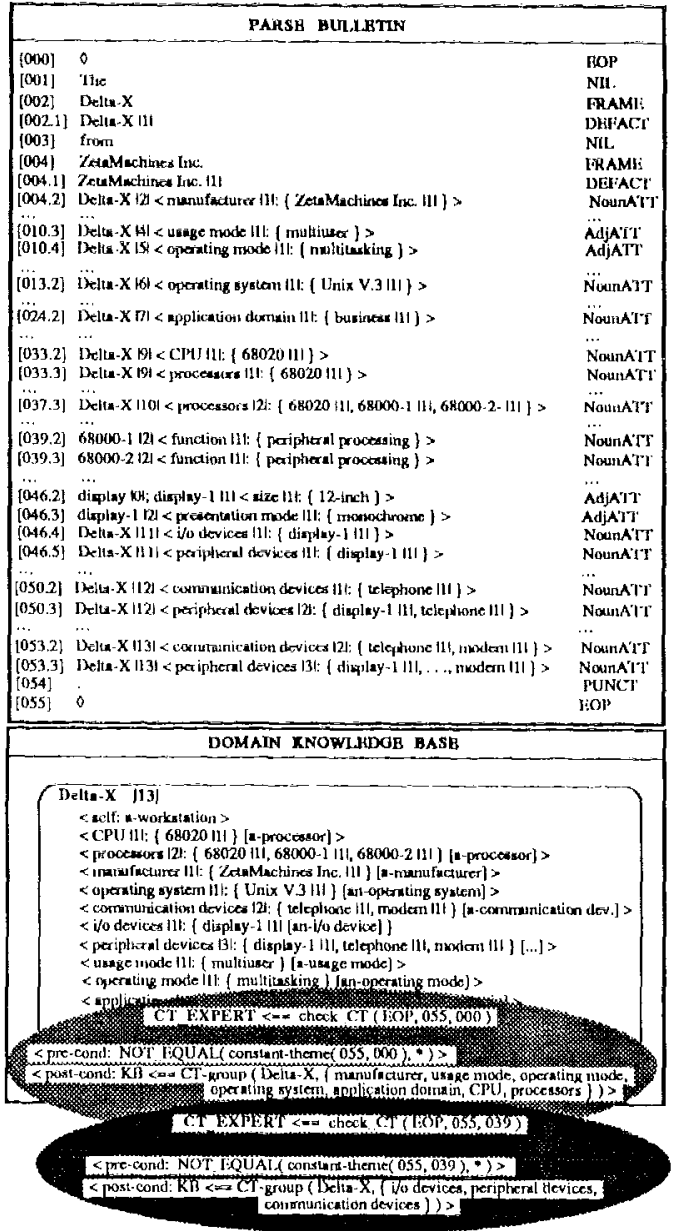

Figure I A Snapstiot of the Parser (also Pre-Conditions Ilolding with respect to a Consfant Theme Pattern)

The DOMAIN KNOWLEDGE BASE (KI for short) contains framc representation structures. Each frame identifier (in bold face) is assigned a list of slots (enclosed by angular brackets). These slots are associated with two different kinds of siot fillers. Permitted slot fillers' are cnclosed in square brackets, [a-frame name], which cluaracterizes the range of possible slot fillers by all those frames which are a subordinate or an instance of frame name. Actual slot fillers are cnclosed in curly braces and can be taken as facts either known a priori to the system or acquired continuously from the text as its understanding proceeds during the parse.

In addition, each concept has attached to it an $\alpha-$ tivation weight counter. The valucs of the weight factors are enclosed by vertical bars attached to each item; if no bars explicitly occur, a zero weight is assumed. Activation weights are incremented (starting from zerolevel activation) whenever a noun denoting its associated concept occurs in the text, and whenever structurebuilding operations in $\mathrm{KB}$ affect that concept. 'The ma- 
nipulation of activation weights serves several purposes, the major one being their use as an indicator of salience of concepts during the text condensation phase, during which text summaries are generated from the text representation structures resulting from the text parse [Reimer \& Hatm 1988].

The text grammar is composed of a sct of distributed grammar experts, each one responsible for some specific linguistic function (c.g., concept attribution via nominal, adjectival or prepositional phrases, anaphora). Each expert is characterized by a unique EXPERT NAME and is activated by a message event, i.c., by receiving a message text which may contain some parameters. In order to check its competence in contributing to the parse, pre-conditions composed of complex test predicates are cvaluated. If these pre-conditions hold for that cxpert, the post-conditions immedialely apply, i.e. messages are sent to qualified actors (to other grammar experts, to the domain $\mathrm{KB}$ or to the bulletin).

\section{A DISTRIBUTED MODEL OF TEXT COHF,RENCE PARSING}

In this paper, we shall not go into the details of phrasal, clausal, and text cohesion parsing (cf. Hahn [1989| for an in-depth consideration of related technical issucs). Instead, we assume that these preliminary activities have already been carried out properly and that some initial structural representation is already available from the bulletin. These requirements are fulfilled in the snapshot of the PARSE BULLETIN in Figure 1, taken after all local parsing events have temintited; this characterizes a state ready to turn to the activation of global text structure compuling experts.

We here consider the end of the paragraph (denoted by the symbol 0 and the class identilier LOP) as an anchoring point for colierence compulation. It is motivated by the observation that -- at least in the sublanguage domain we are currently working in -- major topic movements occur predominantly at paragraph boundaries. This coincides with linguistic evidence for the (text)grammatical status of paragraphs [Ilinds 1979), Giora 1983b, and Zadrozny \& Jensen 1991]. Therefore, the proper recognition of textual macro structures is al ways initialized at the end of a paragraph.

\subsection{Considering Constant Theme}

Constant theme is a colverence pattern which is characterized by inultiple occurrences of a single frame in the PARSE BULLETIN within one paragrapl. Most of its occurrences, in turn, are accompanied by a slot and/or slot filler indicating that some knowledge base operation with respect to frame has been carricd out in $\mathrm{KB}$ (e.g., slot filling as indicated by NounATT or AdjATT for which we shall introduce the LC* descriptor as a convenicnt strorthand notation). It is the continuous claboration of that particular concept that makes the corresponding text passage coherent. While the bulletin maintains the sequential order of these operations, $\mathrm{KB}$ provides the conceptual background for continuous refcrences to the same frame object.

Figure 2 vistalizes the description for constant theme; the DOMAIN KNOWLEDGE BASE window displays all propertics of frame dealt with in a text (passage) in the shadowed arca of the frame box, while those not mentioned in the text are in the remaining white part. Consequently, it is neither necessary that all slots of a frame available in the knowledge base be referred to in the text (as with slot $t_{n+1}, \ldots$, slot $t_{m}$ ), nor that there be any ordering constraint relating single slots of a frame in $\mathrm{KB}$ to the sequence of slot filling operations in the PARSE BULLETIN.

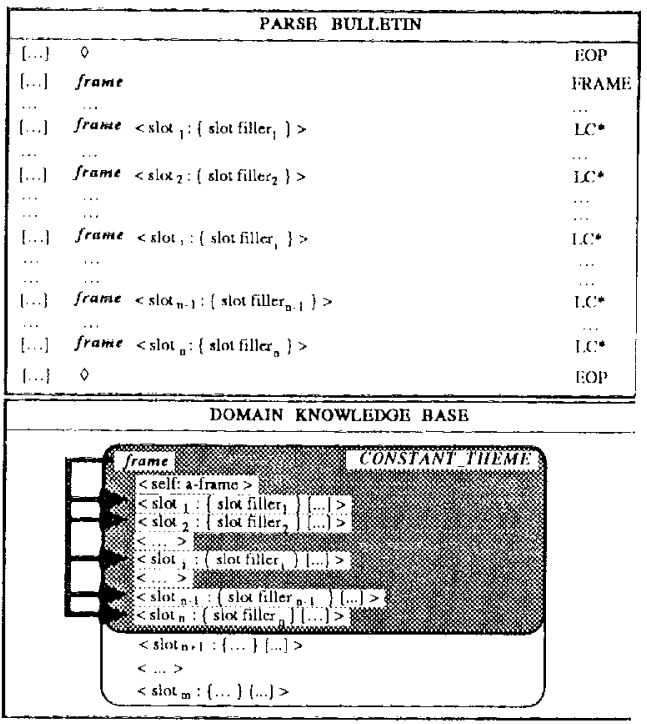

Figure 2 The Cieneral Constant Theme Configuration Patern

The general pattem from Figure 2 is already present in lïgure 1. This contains a description of the parsing results of the first paragraph of text ['T 1.1]. The entries in the PARSE BULLETIN have been worked out by experts for linguistic phenomena on the local Jevel of phrasal, sentence and text cohesion analysis. For the purpose of constant theme computation, we need only consider those entries whose parse descriptor designates manipulations of slots or slot values of some frame (LC*-type descriptors, such as NounATT or Adj$\mathrm{AT} \mathrm{T})$. Other descriptors are irrelevant here and have been left out on purpose in Figure 1. From this we construct the set THEMES. It consists of triples ( frame, slot, bullpos ) where frame is the name of a frame, and slot is the name of a slot of that frame, both co-occurring as lexical parameters of some parse tuple in the PARSE BULLETIN with a LC*-Lype parse descriptor; bullpos gives the parse point in the PARSE BULLETIN where frame and stot occur instantancously. With respect to Figure 1 THEMES is given by:

\footnotetext{
THEMES $=\{$ ( Deloa $X$, manufacturer, $(0) 4)$,

(Della $X$, usage mode, 010$)$,

(Delta.X, operating mode, 010$)$,

(Della-X, operating system, 013 ).

(Dela $X$, application domain, 024 ),

(Della-X, CPU, 033),

(Detha $X$, processors, 033 ),

(Delta-X, processors, 037 ),

(68000-1, function, 039).

(6800) 2, function, 039 ).

(display-1, size, 046),

(display-1, presentation mode, 046),

(Della-X, i/o devices, 046),
} 
(Delta-X, peripherul devices, 046 ).

(Delta-X, communicalion devices, 050),

(Delta-X, peripheral devices, 050 ),

(Della-X, communication devices, 053 ),

(Delia-X, peripheral devices, 0.53 ) )

When considering ITleMLs, we want the criterion for constant theme to be specilied in a way that accounts for the fact that up to parse point '(03\%' each slet (value) manipulation refers to one particular thene (Delta- $X$ ). Between parse point ' $(03)^{\prime}$ ' and ' $(046$ ' there is a minor thematical distortion in that there is no proper reference to that theme, although slots are mentioned which are associated with other concepts. Illowever, from parse point '(46' onward the already established theme is taken up again till the end of the paragraph. In conclusion, Delta- $X$ seems to be a proper candidate for consideration as a constant theme of that paragrajpi. ${ }^{1}$

Figure 1 provides at snapshol of the pre-conditions that are encountered by the CT EXPERT, the coherence expert for ConstartThene. Rurning twice, supplied with different paraneters, it works out the results alluded to above. The grammatical knowledge needed for the determination of a constant theme is incorporated in its pre-condition part. This expression is evaluated TRUE iff constant-theme produces somc theme and an associated non-empty set RHEMES related to theme, otherwise it is FALSE. The conditions for a cont. stant theme can now ix stated more precisely:

\section{constant-theme (textpos, testpos)}

$$
\approx \text { ( theme, RHEMES, newpos ) iff }
$$

(a) testpos < textpos \&

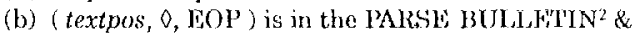

(c) (prepos, $0, \mathrm{EOP}$ ) is also in the PALSH' BUILl: TIN such that prepose textpos and such that no other triple with ' $O$ ' as text item intervenes between prepos and textpos in the PARSI' BUTIJI'TIN \&

(d) newpos $\in[\max ($ prepos, testpos $)+1$, textpos-1]\&

(e) theme is a frame in the DOMAIN KNOWLEIME BASF \&

(f) $\vee \mathrm{k}_{\mathrm{i}} \in[\max ($ prepos, testpos )+1, rewpos-1]:

$$
\text { (theme, slot, } \mathrm{k}_{\mathfrak{i}} \text { ) \& 'THLMLS }
$$

$$
\therefore \rightarrow \text { slot a RHEMES \& }
$$

(g) $-\exists \mathrm{k}$ e $\mid \max ($ prepos, testpos $)+1$, newpos-1|:

(a) alt theme (distinct from theme) is a frame in the DOMAIN KNOWLEDGE' BASL \&

( $\beta$ ) (alt theme, slot, $\mathrm{k}^{\prime}$ ) \& THEMFs \&

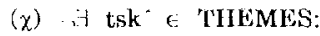

$$
\mathrm{tsk}^{\prime}=\left(\text { theme, slot, } \mathrm{k}^{\prime}\right) \&
$$

(h) $\mid$ RHEMES $\mid>2$ \&

(i) newpos is maximal in the sense that - 1 pos \& $\mid \max ($ prepos, lestpos )+1, textpos-1|: $\Lambda$ pos $>$ newpos \&

conditions (c) - (g) apply, too.

\section{Otherwise, constant-therne (textoos, testros $)=*$}

1 Clearly, this discussion stiould not be taken such that the formal characteriza. tion given betow mly holds for the specific sample text referred to throughou

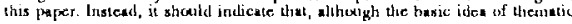
progression pattems is owerwhelmingly simple, ichtife tex/s teso to br less homogereous with respect to these pallemis than one may coisider under clems latwaratory conditions. Thus, formal dexcriptions have to be inherestly robust tow ards kuch locel forms of digrexsions.

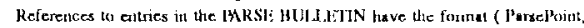
lamélupte, Parseleneriptor).
Sone contunents related to this specification:

(a) The parametcis supplicel to constant-theme span the spatial exicnsion in PALSY RULL,ETIN which is searched for a constan theme; textyos always denotes the end of the current paragraph, i.e. the "1pper bound of the search area, while testpos delimits its lower lound.

(b) The parse poin characterized by textpess inust conbain the cod-of-paraglitph syinbol 0 .

(c) Since testpos niay be any arbitrary parse point pre ceding extpos, prepos denoles the parse point in PARSI: BUII IETIN that contains the end of paratgrayh symbel occurring right lefore the one on parse point textpos.

(d) Alter fixing the search interval in the bulletin for which a constant theme is going to be computed, newpos allows for various choices as to how far at constant theme may actually extend in that interval.

(c) theme may be any frame from $\mathrm{KB}$.

(1) A theme is related to its various shemes according 10 the following condition! at cach bulletin position $\left(k_{i}\right)$ where theme occurs in Tlft:Mls within the in. terval delimited by newpos, its associated stot (singhe thene) is: atsigned to the set RIITMLS.

(g) To guarantec that theme is the only topic dealt with in the text, we atso require that no alt theme different from theme occur in the chosen interval such that it also fonns part of 'THEMES -. $(x)$ accounts for more complicated cases where both, alt theme and theme, may occur at the same parse point.

(h) To rule out insigniticant occurrences of theme the cardinality of RIEMES nuust exced a certain level.

(i) The maximality criterion for newpos rules out choosing too small values of newpos.

l.et us now consider an example of the computation processes involved in actual coherence parsing (sec Figure 1). Various collerence experts start execution ujon consumption of the $\diamond$ synutyol (indicating the end of a parapraph) by the adminisfration expet of the parser, but we shath limit our attention to C'I HXI'ERT (since the others will eventually stave). After receiving

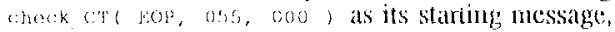
constant-lhente is supplicd with initial parameters: textpos :- 055 , tesipos $=0$ (0)0. Obviously, peepos : 000 , since the analysis starts for the first paragratph of the text. newpos may now range from ' 00$)^{\prime}$ to '(0.54'. Let us consider Delta- $X$ ats theme. (This is a proper choice. If improper choices were minde, constanttheme would not produce a significant result.). The choice for newpos must accominodate the temporary breakdown of the selected theme beginning from posi-

tion ' 039 ', since we have $k$ ' $=0.39$ e $[0001,054]$ wilh alt theme $:=68000-1$ (or $08000-2)$ in THEMLS and no projer triple (Delta- $X$, slot, 039) as required by condition $g(x)$ atove. So newpos las to be adjusted properly to the parse point '(63)', at which point the constant theme pattem for Delta-X eventually terminates for the first time. This produces:

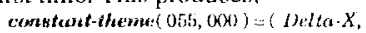

(manufacturer, usagie mode, operating mole, operating sys-

teni, application dorrair, CPU, processors 1039 )

and C'I HXPER'T issues a ("I'-group reatding to KIs incorporating the constam thene logether with its asso-ciated rhemes.

Since the PARSE BUH ITIN has not exhatustively been investigated with respect to its coherence data 
(newpos $+1<$ textpos), CT_EXPERT resumes execution, now starting with a second set of parameters: textpos $=055$, testpos $=039$ (sce the second expert placed into the foreground in Figure 1). Again, prepos $=000$, but due to the new testpos parameter newpos is now in the interval $[40,54]$. The cvaluation of cont. stant-theme $(055,039)$ starts with a proper choice of newpos $=054$. testpos 1 excludes 68000-1 (68000-2) from further consideration. Finally, we obtain

constant-theme 055,039$)=($ Delta-X,

\{i/o devices, peripheral devices, communication devices\}, 054 Note that the occurrence of display- $I$ at parse point '046' does not conflict with criterion (g), since we also have Delta- $X$ (thematically related to ilo devices and peripheral devices) at that parse point (cf criterion $\mathrm{g}(\chi)$ ). Since the end of the paragraph has been reached, the coherence computation process halts.

Figure 3 represents the effects of grouping a constant theme and the rhemes referred to in the text passage (cf. [055.1] and [055.2]) by the shadowed area of the (frame) box. This indicates that the grouped items are treated coherently in a text passage.

\begin{tabular}{|c|c|c|}
\hline & PARSB BULLETTIN & \\
\hline$[000]$ & 0 & EOP \\
\hline .... & $\ldots$ & $\cdots$ \\
\hline$[055]$ & 0 & EOP \\
\hline [055.1] & 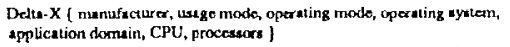 & $\mathrm{Cl}$ \\
\hline [055,2] & Delu-X \{ ito devica, poripheral devices, communication devicas $\}$ & CT \\
\hline \multicolumn{3}{|c|}{ DOMAIN ENOWLADOE BASB } \\
\hline & 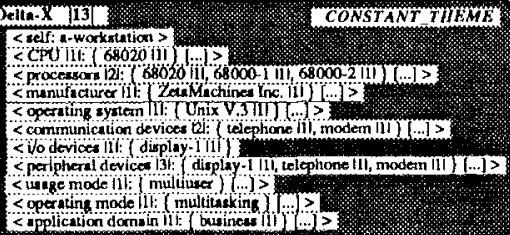 & \\
\hline & $\begin{array}{l}\text { <epplicetion uotware: [ar-application software] > } \\
<\text { price: [A-price]> } \\
\langle\ldots>\end{array}$ & \\
\hline
\end{tabular}

Figure_3 Post-Conditions Holding with respect to a Constant Theme Pattern

\subsection{Remarks on Continuous Thematization of Rhemes and Derived Theme}

Similarily, formal descriptions have been worked out for the other two basic text coherence patterns mentioned above. Instead of a full treatment, we give two rather informal sketches of the underlying regularities as they have been incorporated into our framework. Continuous thematization of rhemes most significantly departs from the constant theme schema just outlined (in fact, both are mutually exclusive) in that the former incorporates a continuous shift of the topics being considered. Figure 4 illustrates this permanent change of issues in a text. The PARSE BULLETIN contains a sequence of local theme-theme pairs with frame $T i$ being the current local theme and slot filler $T$, being its associated local rheme. Text coherence is due to the fact that the current local rheme (slot filler $T_{i}$ ) becomes the next local theme $\left(\right.$ rame $\left._{T i+1}\right)$. This theme-specific connectivity criterion is stressed by the double-sided black arrows in the DOMAIN KNOWLEDGE BASE which link the immediately preceding rheme to its identical theme succes- sor, while local theme-rheme connections are indicated by the one-sided grey arrows which go from the local theme to its associated local rheme. A sequence of local theme-rheme pairs fulfilling the theme-specific connectivity criterion in terms of overlapping paraneters (current rheme becomes next theme) constitutes what is here called continuous thematization of rhemes, i.e. a global theme-theme cluster.

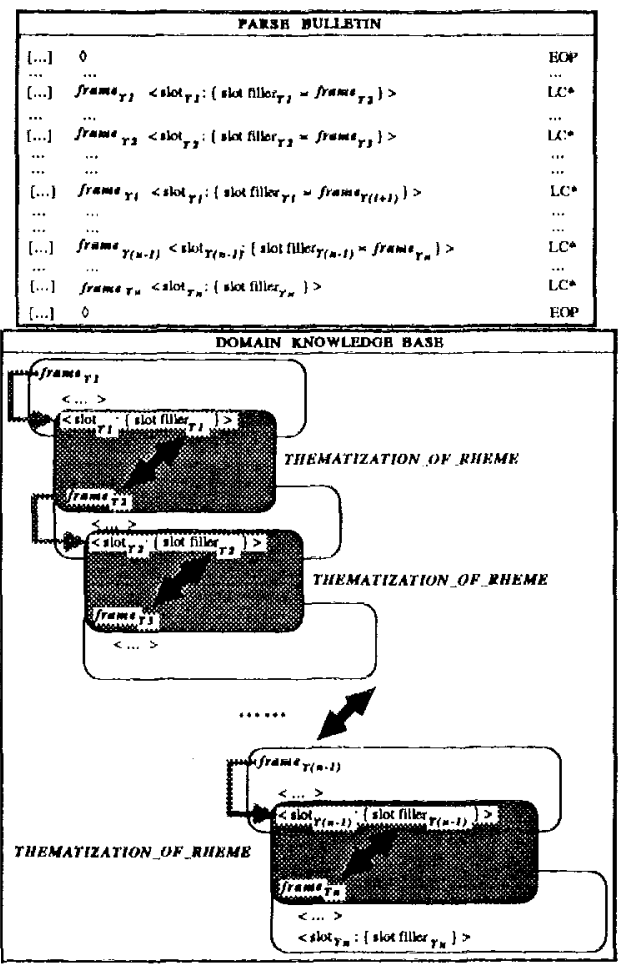

Figure.. 4 The General Continuous Thematization of Rhemes Configuration Pattern

An illustration is given by text fragment [T1.2] in section 3 where bold italics stress the emerging global theme-rheme cluster constituted by the following sequence of overlapping local theme-theme pairs:

Delta-X - manufacturer-ZetaMachines Inc.,

ZetaMachines Inc. - product-Gamma-Z,

Gamma-Z -architecture-Conn. Machine architecture, Conn. Machine architecture-developer-D. Hillis

The third pattem further generalizes the results of the afore-going coherence computations on the paragraph level and extends them over various (adjacent) paragraphs and possibly over the whole text. Consider a series of paragraphs, each one dealing exclusively with one special topic (see Figure 5 below). The first paragraph deals with frame $_{T}$, the second one elaborates on frame $e_{T 2}$, etc. A derived theme can be computed when all these different (sub)topics can be linked to the most specific general (super)topic $\left(\right.$ frame $\left._{T}\right)$. In technical terms, these subtopics are all instances of that 
supertopic.Text [T2] illustrates this phenomenon: thee are three paragraphs whose major topics are Delta-X, Gamma-Z, and Sigma-P; a conceptual generalization step links them to the derived theme workstation. In Figure 5 this relationship is indicated by the arrows pointing from each subtopic (of a single paragraph) to its supertopic, thematically characterizing these paragraphs on a more general level of conceptualization.

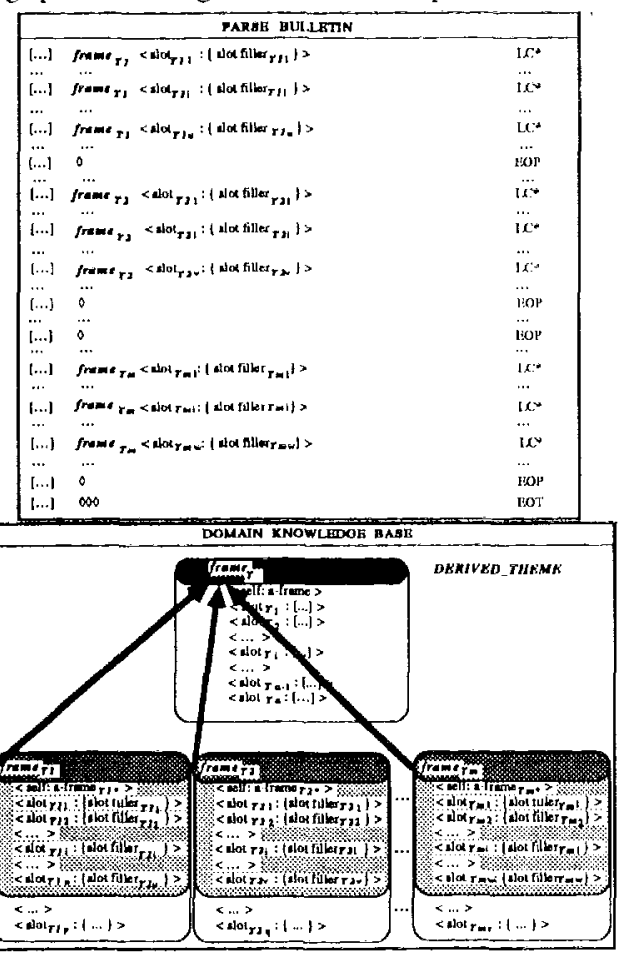

Figure_5 the General Derived Theme Configuration Pattern

5.3 The Merits of Text Coherence Parsing Among the many advantages to having text cohenence phenomena under computational control we here emphasize their potential for information retrieval dialogs. Evidence for this comes from our experiments with TOPOGRAPHIC, an interactive graphical interface to TOPIC's text knowledge bases [Thiel \& Hammwöhner 1987]. In particular, we observed a close functional relationship between the selection of particular coherence patterns and particular search states during the retrieval process which is performed on network representations of text summaries, so-called text graphs:

1) Constant Theme coherently characterizes a variety of facts related to one particular topic. A CT-based search operation enhances the user's knowledge of that topic by presenting facets (or data related to those facets) the user is probably not aware of, although they may be relevant to the solution of his or her problem.

2) Continuous Thematization of Rhemes links a set of formerly unrelated topics by a coherent line of conceptual dependencies (curnent rheme becomes next theme). A CTR-based search operation therefore provides the basis for thematical associations and stim- ulates previously unconsidered lines of reasoning by thematically constrained browsing.

3) Derived Theme groups hicrarchically related topics and thus may enhance the knowledge of alternatives of the particular topic (and facts related to it) under focused attention of the user (by way of stimulating comparisons, ncognizing information gaps, etc.).

\section{FINAL REMAKKS}

In this paper, a structural model of text coherence computation has been proposed that strongly exploits the knowledge chunking inherent to frame representations. These precompiled knowledge structures are instantiated by the topical evolution of a text as represented in the parser's bulletin. Thus, various coherence phenomena can be distinguished by particular instantiation patterns

7 constant theme is defined by multiple instantiations of aggregation (or conceptual association) relations for one particular frame item in $\mathrm{KB}$;

$\square$ continuous thematization of rhemes is defined by multiple instantiations of aggregation relations for continuously changing, though locally overlapping frame items in $\mathrm{KB}$;

7 derived theme is defined by multiple instantiations of generalization/classification relations holding between subparts of a frane hierarchy in $\mathrm{KB}$.

A more elaborated formal description of this nodel - including those parts which could only be treated rather sketchily in this contribution - is given in llahn [1991]. The parser is currently runining on SUN SPARCStations under Unix (SUNOS V4.1.1). The functionality described in this paper is fully operational and part of the TOPIC text understariding system.

\section{REFEKRNCES}

Alterman, R. [1982]. A syaiem of seven coherence ralations for hierarchically organizing evert concepts in text. Univ, of Texax at Auscin (TR-188)

Brachmen, R.J.: V.P. Gll bert: III. Levengue [1985]. An easential hybrid reatoning ayrters knowled Ba and symbol level sccounts of Kryptom. Proc. IJCA/ 85, pp. 532.539.

Danex, fi [ 1974 ]. Functional antatce perdpertive and the organization of the text In IF Danes, ed. Popers on functional sentemse perspective. Acedemin, $106-128$.

van Dijk, J', A. [1980]. Mocrasiructures. Hillidale/N]: i. Exlbetun.

Glora, R. [19838]. Segmentution and segnent cohesion: on the thenatic organiza lion of the lext. Text, $3(2): 155-181$

Glora, R. [1983b]. I unctional paragraph perspective. If J. Pedobfi \& J: Sözec, cais Micro and macro connexity of iexit. Hentairg: H. Bluske, pp. 153-182.

Ilahn. U. [1989]. Making uiderstanders out of parers. Iniermational Jourmal of Inielligent Systems. 4(3): 345-393.

Ilatur, []. [1990]. Lexikalisch verteilles Jext-Parsing. Herlin: Springer

ILhn, U. [1991]. Distributed text structure parsing. I inguilische Informatik Komputerlinguistik. Univ. Freiburg. CIJF-Report 491 .

flindu, J. $[1979]$. Orgatizational puttems in discounte. In 'f. Givón, ed. Syntax and semantics, Vol. 12. New York/NY: Acadertic Pr, pp.135-157.

llobba, J. K. [1982]. Tawards an undertending of coberence in discoursc. II W.G Leiner \& M. Ringle, eds. Strategies for molural language processing. Hillsdaled NJ: L, Litraum, pp.223-243.

KIntuch, W; T.A. Vun IHJk [1978]. Toward a model of text comprehession soul productiont. Psychological Revirw, 85(5): 363.394.

Kurzos, D. [1984] Themes, hypertiemes and the discourse structure of bitiah legal texts. Toxt, 4(1-3):31-55

Mann, W.C.; S.A. Thompson [1988]. Khetorical structure theory: towards funclional theory of text organization. Tiext, B(3): 243-287.

McKeown, K. [1985]. Dixcoure strategice for generating rintural-lungunge text Arificial Intalligence, $27(1): 1-41$

Puatejoviky. J. [1987]. An integrated theory of discourse anslysis. In S. Nirenbirg, ed. Machine translation. Cumbridge: Cembridge U.P, pp.168-191.

Relchman, R. [1978]. Comtersulional coherency. Cognitive Science, 2(4): 283-327.

Kalmer, U.; U. Indin [198B). Text condenation as knowledge basc abstraction. Pnoc. Aih cons. on artifitial invelligunce applications (CANA-8B), pp.338-344,

Rumethart, D.L. [1975]. Notes on a schema for stories. In D. Bobrow \& A. Col. lins, eds. Representation and understanding. New York: Acodemic P., 211- 236.

Schn, R.; L. Polnnyl [1988]. An angmented context free grammex for discourse Proc. GOLJNG88, pp.573-5\%

Schank, R.C.; M. Lebowlty; L Btrnbeum [1950]. An integrated understander. Amirican Jowrmal of Computationol Linguistics, 6(1): 13-30.

Thlel, U.; R. Hammothner [1987) Infonmationsl zooning: in interaction model for the graphical acceas to lext knowlodge buses. Proc. JOth ACM SIGIR cony. on research \& development in information retriaval. pp. 45-56

Tucker, A. B.; NIrenburg, S; Rakkin, V. [1986]. Discesure and cotsesion in ex pexitory text. Proc. COLING; 86, pp.181-183.

Zadrozmy, W. Jensen, K. [1991]. Semantick of paregraphe. Compisational Linoustics. $17(2): 171.209$. 